\title{
Inhibition of thermohaline mixing by a magnetic field in Ap star descendants: implications for the Galactic evolution of ${ }^{3} \mathrm{He}$
}

\author{
C. Charbonnel ${ }^{1,2}$ and J.-P. Zahn ${ }^{3}$ \\ ${ }^{1}$ Geneva Observatory, University of Geneva, 51 chemin des Maillettes, 1290 Sauverny, Switzerland \\ e-mail: Corinne.Charbonnel@obs . unige.ch \\ ${ }^{2}$ Laboratoire d'Astrophysique de Toulouse et Tarbes, CNRS UMR 5572, Université Paul Sabatier Toulouse 3, \\ 14 Av. E. Belin, 31400 Toulouse, France \\ 3 LUTH, Observatoire de Paris, CNRS, Université Paris-Diderot, Place Jules Janssen, 92195 Meudon, France \\ e-mail: Jean-Paul.Zahn@obspm.fr
}

Received 26 September 2007 / Accepted 30 October 2007

\section{ABSTRACT}

\begin{abstract}
Aims. To reconcile the measurements of ${ }^{3} \mathrm{He} / \mathrm{H}$ in Galactic $\mathrm{HII}$ regions with high values of ${ }^{3} \mathrm{He}$ in a couple of planetary nebulae, we propose that thermohaline mixing is inhibited by a fossil magnetic field in red giant stars that are descendants of Ap stars.

Methods. We examine the effect of a magnetic field on the salt-finger instability, using a local analysis.

Results. We obtain a threshold for the magnetic field of $10^{4}-10^{5}$ Gauss, above which it inhibits thermohaline mixing in red giant stars located at or above the bump. Fields of that order are expected in the descendants of the Ap stars, taking into account the contraction of their core.

Conclusions. We conclude that in a large fraction of the descendants of Ap stars thermohaline mixing does not occur. As a consequence these objects must produce ${ }^{3} \mathrm{He}$ as predicted by the standard theory of stellar evolution and as observed in the planetary nebulae NGC 3242 and J320. The relative number of such stars with respect to non-magnetic objects that undergo thermohaline mixing is consistent with the statistical constraint coming from observations of the carbon isotopic ratio in red giant stars. It also satisfies the Galactic requirements for the evolution of the ${ }^{3} \mathrm{He}$ abundance.
\end{abstract}

Key words. instabilities - stars: abundances - stars: evolution - Galaxy: evolution - magnetic fields

\section{Introduction}

Numerous observations provide compelling evidence for a noncanonical mixing process which modifies the surface abundances of $\mathrm{Li}, \mathrm{C}$ and $\mathrm{N}$ of low-mass red giants ${ }^{1}$ when they reach the bump in the luminosity function (see references in Charbonnel \& Zahn 2007, hereafter CZ07). The quest for a mechanism which could lead to that extra-mixing between the hydrogen-burning shell and the convective envelope of red giant branch (RGB) stars has initially been unsuccessful. For example rotational mixing, i.e. rotation-induced meridional circulation and shear turbulence (Zahn 1992; Maeder \& Zahn 1998), is insufficient to explain the observed abundance pattern, as was shown by Charbonnel \& Palacios (2004) and Palacios et al. (2006). But recently Eggleton et al. (2006) suggested a possible cause of such mixing, namely the molecular weight inversion created by the ${ }^{3} \mathrm{He}\left({ }^{3} \mathrm{He}, 2 \mathrm{p}\right){ }^{4} \mathrm{He}$ reaction in the upper part of the hydrogen-burning shell. Based on 3D hydrodynamic simulations of a low-mass star at the RGB tip (Dearborn et al. 2006), they found that such a $\mu$-profile is unstable and produces very efficient mixing. They claimed that this mixing was due to the well-known Rayleigh-Taylor instability, which occurs in incompressible fluids when there is a density inversion. In stellar interiors, which are stratified due to their compressibility, a

${ }^{1}$ I.e., stars with initial masses below $\sim 2-2.5 M_{\odot}$ which evolve along the Red Giant Branch (RGB) to high luminosities until helium is ignited in their core under degenerate conditions. similar dynamical instability occurs when the Ledoux criterion for convection is satisfied (Ledoux 1947), but it acts to render the temperature gradient adiabatic rather than to suppress the density inversion. Presumably it is this instability that Eggleton and colleagues observed with their 3D code, as attested by the high velocities they quote, because they started their simulation with a unrealistic 1D model which did not include any mixing, in which the $\mu$-inversion had already attained its maximum value.

In reality, the first instability to occur in a star, as the inverse $\mu$-gradient gradually builds up, is the thermohaline instability, as was pointed out by CZ07. This double-diffusive instability is observed in salted water in the form of elongated fingers, when the temperature is stably stratified, but salt is not, with fresh water at the bottom and salted at the top, the overall stratification being dynamically stable (Stern 1960).

It was Ulrich (1972) who first noticed that the ${ }^{3} \mathrm{He}\left({ }^{3} \mathrm{He}, 2 \mathrm{p}\right){ }^{4} \mathrm{He}$ reaction would cause a $\mu$-inversion, and he was the first to derive a prescription for the turbulent diffusivity produced by the thermohaline instability in stellar radiation zones. This prescription is based on a linear analysis, and it is certainly very crude, but it has the merit to exist. When it is applied to the $\mu$-inversion layer in RGB stars, with the shape factor recommended by Ulrich and sustained by laboratory experiments (Krishnamurti 2003), it yields a surface composition that is compatible with the observations of the carbon isotopic ratio as well as of the abundances of lithium, carbon and nitrogen in RGB stars (Fig. 3 of CZ07). 
Simultaneously thermohaline mixing leads to the destruction of most of the ${ }^{3} \mathrm{He}$ produced during the star's lifetime (Fig. 4 of CZ07). It seems thus that we have finally identified the mechanism responsible for abundance anomalies in RGB stars, which simultaneously accounts for the measurements of ${ }^{3} \mathrm{He}$ in Galactic HII regions (Balser et al. 1994, 1999a; Bania et al. 1997, 2002) $)^{2}$.

A problem remains however, namely that all RGB stars brighter than the bump should undergo thermohaline mixing since they develop the same $\mu$-inversion. As underlined by Balser et al. (2007), this was obviously not the case at least in two stars, i.e., in the RGB progenitors of the Planetary Nebulae NGC 3242 and J320. Slightly more massive than the Sun, these $\mathrm{PNe}$ are indeed caught in the act of returning to the interstellar matter fresh elements synthesized in their womb among which ${ }^{3} \mathrm{He}$ with the amount predicted by classical stellar models (Balser et al. 1997, 1999b, 2006; Galli et al. 1997). On the other hand the carbon isotopic ratio data indicate that a few RGB stars escape that extra-mixing - about $4 \%$ according to Charbonnel \& do Nascimento (1998). This is consistent with the predictions of the chemical evolution models, which suggest that fewer than $10 \%$ of all planetary nebulae enrich the ISM with ${ }^{3} \mathrm{He}$ at the level of classical stellar models (Galli et al. 1997; Tosi 1998, 2000; Palla et al. 2000, 2002; Romano et al. 2003), thus supporting the self-consistency of the extra-mixing mechanism.

It is tempting to confront these numbers with the fraction of the Ap stars (suspected to all host magnetic fields) relative to all A stars, which is $5-10 \%$ as estimated by Wolff (1968). Since A-type stars are the progenitors of the more massive RGB stars, we are led to conjecture that in those giants that show no sign of extra-mixing, the thermohaline instability is inhibited by a deeply buried magnetic field.

Here we check that possibility by examining the effect of a magnetic field on the salt-finger instability, using a simple local analysis.

\section{Thermohaline instability in the presence of a magnetic field}

All perturbations (displacement $\boldsymbol{\xi}$, magnetic field $\boldsymbol{b}$, pressure $P^{\prime}$, temperature $T^{\prime}$, molecular weight $\mu^{\prime}$ ) are expanded in Fourier modes

$\exp \left[\mathrm{i}\left(k_{x} x+k_{y} y+k_{z} z\right)+s t\right]$

where we use local Cartesian coordinates, with the $z$-axis pointing in the vertical direction.

We begin by deriving the buoyancy force, which is weakened through radiative damping; we neglect all other forms of dissipation that operate more slowly: Ohmic, molecular diffusion, viscosity. We split the buoyancy frequency in two parts, the first due to the thermal stratification and the second to the composition gradient:

$N^{2}=N_{t}^{2}+N_{\mu}^{2}=\frac{g \delta}{H_{P}}\left(\nabla_{\mathrm{ad}}-\nabla\right)+\frac{g \varphi}{H_{P}} \nabla_{\mu}$

\footnotetext{
${ }^{2}$ According to classical theory of stellar evolution which predicts a large production of ${ }^{3} \mathrm{He}$ by low-mass stars, Galactic HII regions should be highly enriched in ${ }^{3} \mathrm{He}$ since they formed out of matter that has undergone 12 billion years of chemical evolution. However, their ${ }^{3} \mathrm{He}$ content is similar to that of the Sun, and thus no evidence for any enrichment of ${ }^{3} \mathrm{He}$ during the last $4.5 \mathrm{Gyr}$ was found. In addition, the "best" determination in a Galactic HII region (namely S209) by Bania et al. (2002) has yielded $\mathrm{a}^{3} \mathrm{He}$ abundance almost identical to the WMAP value, indicating that the stellar contribution to ${ }^{3} \mathrm{He}$ is very small.
}

with the usual notations, and

$\nabla_{\mu}=\frac{\mathrm{d} \ln \mu}{\mathrm{d} \ln P} \quad \delta=-\left(\frac{\partial \ln \rho}{\partial \ln T}\right)_{P, \mu} \quad \varphi=\left(\frac{\partial \ln \rho}{\partial \ln \mu}\right)_{P, T}$.

We shall consider here only the case where the stratification is dynamically stable $\left(N^{2} \geq 0\right)$, i.e. when the Ledoux criterion for stability is satisfied:

$\nabla_{\mathrm{ad}}-\nabla+\left(\frac{\varphi}{\delta}\right) \nabla_{\mu}>0$

The linearized heat equation may be written as

$s \frac{T^{\prime}}{T}+\frac{N_{t}^{2}}{g} s \xi_{z}=-\kappa k^{2} \frac{T^{\prime}}{T}$

where $k^{2}=k_{x}^{2}+k_{y}^{2}+k_{z}^{2}=k_{\mathrm{h}}^{2}+k_{z}^{2}$ and $\kappa$ is the thermal diffusivity. (We simplify the Laplacian by assuming that $\kappa$ does not vary much over one vertical wavelength.) Likewise, the advection equation for the molecular weight perturbation takes the form

$s \frac{\mu^{\prime}}{\mu}-\frac{N_{\mu}^{2}}{g} s \xi_{z}=0$.

Thus the buoyancy force is given by

$-g \frac{\rho^{\prime}}{\rho}=g\left(\frac{T^{\prime}}{T}-\frac{\mu^{\prime}}{\mu}\right)=-\left[\frac{N_{t}^{2}}{1+\kappa k^{2} / s}+N_{\mu}^{2}\right] \xi_{z}$.

Turning next to the Lorentz force, we perturb the magnetic field $\boldsymbol{B}_{0}=\left(B_{x}, 0, B_{z}\right)$ by the displacement $\boldsymbol{\xi}$, and draw the field perturbation $\boldsymbol{b}$ from the induction equation:

$\boldsymbol{b}=\boldsymbol{\nabla} \times\left(\boldsymbol{\xi} \times \boldsymbol{B}_{0}\right)$

It is then straightforward to calculate the perturbation of the Lorentz force per unit volume:

$\boldsymbol{L}=\frac{1}{4 \pi \rho}\left[(\boldsymbol{\nabla} \times \boldsymbol{b}) \times \boldsymbol{B}_{0}\right]$,

where we neglect the variation of $\boldsymbol{B}_{0}$ over a perturbation wavelength. From here on we drop the index 0 designating the basic field.

It remains to implement the buoyancy and Lorentz forces in the equation of motion, which we simplify by neglecting the effect of rotation,

$s^{2} \boldsymbol{\xi}+\left[\frac{N_{t}^{2}}{1+\kappa k^{2} / s}+N_{\mu}^{2}\right] \boldsymbol{e}_{z} \xi_{z}=-\mathrm{i} \boldsymbol{k} \frac{P^{\prime}}{\rho}+\boldsymbol{L}$.

Introducing the Alfvén velocity $\boldsymbol{a}=\boldsymbol{B} / \sqrt{4 \pi \rho}$, we get

$$
\begin{aligned}
& \mathrm{i} k_{x} \frac{P^{\prime}}{\rho}+\left[s^{2}+a_{z}^{2}\left(k_{x}^{2}+k_{z}^{2}\right)\right] \xi_{x} \\
& \quad+k_{y} a_{z}\left(k_{x} a_{z}-k_{z} a_{x}\right) \xi_{y}-k_{x}^{2} a_{x} a_{z} \xi_{z}=0, \\
& \mathrm{i} k_{y} \frac{P^{\prime}}{\rho}+k_{y}\left(k_{x} a_{z}-k_{z} a_{x}\right)\left(a_{z} \xi_{x}-a_{x} \xi_{z}\right) \\
& \quad+\left[s^{2}+\left(k_{x} a_{x}+k_{z} a_{z}\right)^{2}+k_{y}^{2}\left(a_{x}^{2}+a_{z}^{2}\right)\right] \xi_{y}=0, \\
& \mathrm{i} k_{z} \frac{P^{\prime}}{\rho}-a_{x} a_{z}\left(k_{x}^{2}+k_{z}^{2}\right) \xi_{x}-k_{y} a_{x}\left(k_{x} a_{z}-k_{z} a_{x}\right) \xi_{y} \\
& \quad+\left[s^{2}+a_{x}^{2}\left(k_{x}^{2}+k_{z}^{2}\right)+\frac{N_{t}^{2}}{1+\kappa s^{2} / \sigma}+N_{\mu}^{2}\right] \xi_{z}=0,
\end{aligned}
$$


which we complete with the continuity equation, written in its simplest form, assuming that the Boussinesq approximation is valid, i.e. that $\left|k_{z}\right| \gg|\mathrm{d} \ln \rho / \mathrm{d} z|$ :

$k_{x} \xi_{x}+k_{y} \xi_{y}+k_{z} \xi_{z}=0$.

The determinant of that fourth-order system is the dispersion relation from which we derive the eigenvalues $s$, i.e. the growthrate of the instability.

In the non-dissipative case, this dispersion relation reduces to (cf. Kumar et al. 1999)

$\left(s^{2}+(\boldsymbol{k} \cdot \boldsymbol{a})^{2}\right)\left(s^{2}+\frac{k_{\mathrm{h}}^{2}}{k^{2}} N^{2}+(\boldsymbol{k} \cdot \boldsymbol{a})^{2}\right)=0$.

The first set of (imaginary) roots corresponds to Alfvén waves, and the second to internal gravity waves modified by the magnetic field.

When thermal dissipation is included, these waves are damped, and a fifth root appears, which yields the growth-rate of the thermohaline instability in the presence of a magnetic field:

$s=-\kappa k^{2} \frac{N_{\mu}^{2}\left(k_{\mathrm{h}} / k\right)^{2}+(\boldsymbol{k} \cdot \boldsymbol{a})^{2}}{\left(N_{t}^{2}+N_{\mu}^{2}\right)\left(k_{\mathrm{h}} / k\right)^{2}+(\boldsymbol{k} \cdot \boldsymbol{a})^{2}}$.

Note that the magnetic field always plays a stabilizing role; it overcomes the destabilizing effect of the inverse $\mu$ gradient when

$(\boldsymbol{k} \cdot \boldsymbol{a})^{2}>-N_{\mu}^{2}\left(k_{\mathrm{h}} / k\right)^{2}$.

We may assume that the horizontal size $\left(\lambda=2 \pi / k_{\mathrm{h}}\right)$ of the ${ }^{3} \mathrm{He}$ fingers is much smaller than their vertical extent: $k_{\mathrm{h}} \gg k_{z}$. Then it is mainly the horizontal component $B_{\mathrm{h}}$ of the field that prevents the thermohaline instability, when

$B_{\mathrm{h}}^{2}>\frac{\rho \lambda^{2}}{\pi}\left|N_{\mu}^{2}\right|$.

This criterion is sufficiently accurate for our purpose, although it has been established assuming that $\left|N_{\mu}^{2}\right|$ does not vary much over a vertical wavelength of the perturbation, a condition which is not fulfilled in the real star.

We are aware that the magnetic field itself may undergo instabilities (see Spruit 1999; Menou et al. 2004) that are not captured by our crude analysis, since we ignored both rotation and the spatial variation of $\boldsymbol{B}_{0}^{3}$. We have thus implicitly assumed that magnetic instabilities, if any, appeared before the inverse $\mu$-gradient built up, and that they led to a stable field configuration.

Figure 1 shows the critical value of $B_{\mathrm{h}}$ predicted by Eq. (15) in the region of $\mu$-inversion of a $1.5 M_{\odot}$ model with solar metallicity. This model is located at the bump in the luminosity function, i.e., where thermohaline mixing should be fully efficient in the absence of magnetic field and where the $\mathrm{C}, \mathrm{N}$ and $\mathrm{Li}$ abundance anomalies show up on the RGB (Charbonnel 1994). Here we assume that the finger width is $1 / 10$ of the height of the $\mu$-inversion region. According to our criterion, a magnetic field of $B_{\mathrm{h}} \approx 10^{5}$ Gauss suppresses entirely the thermohaline mixing, while a field of $10^{4} \mathrm{G}$ is sufficient to inhibit the instability in the upper $1 / 3$ of the $\mu$ inversion domain. We also computed (not shown here) the critical value of $B_{\mathrm{h}}$ at the bump in

${ }^{3}$ Busso et al. (2007) suggested that such instabilities might be the cause of extra-mixing. In that case however, the presence of deeply buried magnetic fields would have to be the rule, rather than the exception. We feel thus that the scarcity of magnetism observed in the A-type stars speaks in favor of the alternate scenario that we present here.

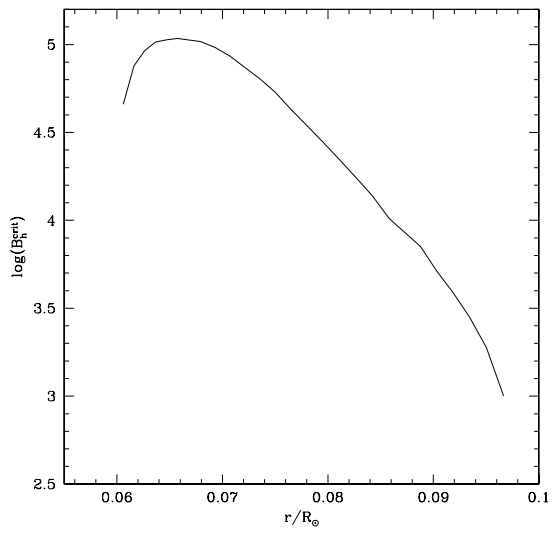

Fig. 1. Critical value of the magnetic field which inhibits thermohaline mixing in a $1.5 M_{\odot}, Z_{\odot}$ model located at the bump.

a $2 M_{\odot}$ model at solar metallicity, and also in a $0.9 M_{\odot}$ model with $[\mathrm{Fe} / \mathrm{H}]=-1.8$. Due to the fact that the structure of the $\mathrm{H}$-burning region is very similar in all the cases, we obtain comparable values for $B_{\mathrm{h}}^{\text {crit }}$.

Magnetic fields of that order are expected in the deep interior of the red giant descendants of the Ap stars. On the main sequence, these Ap stars possess surface fields of a few $10^{2}$ to about $3 \times 10^{4} \mathrm{G}$, which are believed to be of fossil origin; if so, the field is deeply rooted and its Ohmic decay time largely exceeds the main sequence lifetime. This scenario seems to be confirmed by a recent investigation which concludes that Ap stars whose mass is less than $3 M_{\odot}$ show no compelling evidence for field decrease during their main sequence life (Landstreet et al. 2007) ${ }^{4}$. The hypothesis of the fossil nature of magnetism in the Ap stars has recently been strengthened by the discovery that their progenitors are certainly the Herbig Ae/Be stars (Wade et al. 2007a,b).

After the main sequence phase, as the central region of the star contracts during evolution on the giant branch, the magnetic field may be enhanced by up to two orders of magnitude, due to flux conservation. Thus it can easily exceed the critical strength that ensures the suppression of the thermohaline instability. We thus consider as plausible that those red giants which are prevented from undergoing the non-canonical mixing are the descendants of main sequence Ap stars, because the magnetic field of fossil origin suppresses in them the thermohaline instability. If this scenario is correct, one should observe magnetic fields in RGB stars that behave classically, and in PNe such as NGC 3242 and $\mathrm{J} 320$.

\section{Discussion}

The observational uncertainties in effective temperature, luminosity and metal content of Ap stars hamper a precise determination of their mass (see Bagnulo et al. 2006). According to a survey covering the Ap/Bp stars located within $100 \mathrm{pc}$ from the Sun (Power et al. 2006), magnetic stars range at solar metallicity between 3.6 and $1.5 M_{\odot}$ (with one exception at $6 M_{\odot}$ ). The lower limit coincides with the appearance of developed surface convection zones, which depends on the initial stellar metallicity. If this correlation held in the past independently of the bulk stellar metallicity, metal-poor magnetic stars could have extended to

\footnotetext{
4 The rapid field decline observed above this mass may be explained by the increased turbulent diffusion occurring in the larger convective core.
} 
lower initial mass: standard models predict indeed that the convection zone of a $0.9 M_{\odot}$ main sequence star with $[\mathrm{Fe} / \mathrm{H}]=-2$ has the same thickness as that of a $1.5 M_{\odot}$ main sequence star of solar metallicity (Talon \& Charbonnel 2004). As a consequence, one can conjecture that below solar metallicity, Ap star counterparts formed with initial masses much below $1.5 M_{\odot}$. This expected dependancy of the mass threshold for the magnetic inhibition of thermohaline mixing with metallicity is of direct relevance to the Galactic evolution of ${ }^{3} \mathrm{He}$ and will have to be taken into account in future chemical evolution models which consider metal-dependant yields.

Galli et al. (1997) have determined a mass of $1.2 \pm 0.2 M_{\odot}$ for the progenitor of the planetary nebulae NGC 3242. Although such a determination is quite a feat and is undermined by several observational and theoretical difficulties, we find very encouraging that the initial mass derived for this object is so close to the lower mass limit for Ap stars at solar metallicity. We have to note indeed that the metallicity of NGC 3242 is half-solar (Barker 1985), so that the lower mass for Ap stars may already be shifted towards a lower value.

It remains to be checked whether the magnetic stars are in sufficient number on the main sequence to account for the $\approx 4 \%$ of red giants which are spared from non-canonical mixing as estimated from the carbon isotopic ratio data (Charbonnel \& Do Nascimento 1998). The classical value of the incidence of Ap/Bp stars, which is $5-10 \%$ based on a magnitude-limited sample (Wolff 1968), has been challenged by the aforementioned survey performed by Power et al. (2006): in the solar neighborhood they find only $1.7 \%$ of magnetic stars. This value is close to that (i.e., $3.5 \%$ ) derived by North (1993) for Ap stars with masses between 1.7 and $2.5 M_{\odot}$ that are members of Galactic open clusters, and by Paunzen et al. $(2006 ; \sim 2.2 \%)$ for chemically peculiar stars in young LMC open clusters. We note that the bulk incidence of Ap stars is very sensitive to the chosen value of the lower mass limit for these objects in a given sample (see Fig. 4 of Power et al. 2006 or Table VII of North 1993). In conclusion, we consider that the present statistics support our suggestion that thermohaline mixing is inhibited by a fossil magnetic field in those red giant stars that are the descendants of main sequence Ap stars. Within this framework we reconcile the long-standing problem of ${ }^{3} \mathrm{He}$ overproduction on the Galactic timescale with the high values of ${ }^{3} \mathrm{He}$ observed in a couple of planetary nebulae. We encourage the observers to look for magnetic fields in "classical" RGB and in NGC 3242 and J320 which we propose to call "thermohaline deviant stars".

Acknowledgements. We thank the referee for her/his helpful comments. C.C. is particularly grateful to D. Balser, T. Bania, C. Chiappini, R. Rood and M. Tosi for enlightening discussions on the "3 $\mathrm{He}$ problem" over the years. We thank J. Landstreet, P. North and G. Wade for their comments on Ap statistics.
C.C. is supported by the Swiss National Science Foundation (FNS). We acknowledge the financial support of Programme National de Physique Stellaire (PNPS) of CNRS/INSU, France.

\section{References}

Bagnulo, S., Landstreet, J. D., Mason, E., et al. 2006, A\&A, 450, 777 Balser, D. A., Bania, T. M., Brockway, C. J., Rood, R. T., \& Wilson, T. L. 1994, ApJ, 430, 667

Balser, D. A., Bania, T. M., Rood, R. T., \& Wilson, T. L. 1997, ApJ, 483, 320

Balser, D. A., Bania, T. M., Rood, R. T., \& Wilson, T. L. 1999a, ApJ, 510, 759

Balser, D. A., Rood, R. T., \& Bania, T. M. 1999b, ApJ, 522, L73

Balser, D. A., Goss, W. M., Bania, T. M., Rood, R. T., \& Wilson, T. L. 2006, ApJ, 640, 360

Balser, D. A., Rood, R. T., \& Bania, T. M. 2007, Science, 317, 1171

Bania, T. M., Balser, D. A., Rood, R. T., Wilson, T. L., \& Wilson, T. J. 1997, ApJS, 113, 353

Bania, T. M., Rood, R. T., \& Balser, D. A. 2002, Nature, 415, 54

Barker, T. 1985, ApJ, 294, 193

Busso, M., Wasserburg, G. J., Nollett, K. M., \& Calandra, A. 2007 [arXiv:0708.2949]

Charbonnel, C. 1994, A\&A, 282, 811

Charbonnel, C., \& do Nascimento, J. D. 1998, A\&A, 336, 915

Charbonnel, C., \& Palacios, A. 2004, IAU Symp., 215, 440

Charbonnel, C., \& Zahn, J.-P. 2007, A\&A, 467, L15 (CZ07)

Dearborn, D. S. P., Eggleton, P. P., \& Lattanzio, J. C. 2006, ApJ, 639, 405

Eggleton, P. P., Dearborn, D. S. P., \& Lattanzio, J. C. 2006, Science, 314, 1580

Galli, D., Stanghellini, L., Tosi, M., \& Palla, F. 1997, ApJ, 477, 218

Krishnamurti, R. 2003, J. Fluid Mech., 483, 287

Kumar, P., Talon, S., \& Zahn, J.-P. 1999, ApJ, 520, 859

Landstreet, J. D., Bagnulo, S., Andretta, V., et al. 2007, A\&A, 470, 685

Ledoux, P. 1947, ApJ, 105, 305

Maeder, A., \& Zahn, J.-P. 1998, A\&A, 334, 1000

Menou, K., Balbus, S. A., \& Spruit, H. C. 2004, ApJ, 607, 564

North, P. 1993, IAU Colloq. 138, ASP Conf. Ser., 44, 577

Palacios, A., Charbonnel, C., Talon, S., \& Siess, L. 2006, A\&A, 453, 261

Palla, F., Bachiller, R., Stanghellini, L., Tosi, M., \& Galli, D. 2000, A\&A, 355, 69

Palla, F., Galli, D., Marconi, A., Stanghellini, L., \& Tosi, M. 2002, ApJ, 568, L57

Paunzen, E., Maitzen, H. M., Pintado, O. I., et al. 2006, A\&A, 459, 871

Power, J., Wade, G. A., Hanes, D. A., Aurière, M., \& Silvester, J. 2006 [arXiv: astro-ph/0612557]

Romano, D., Tosi, M., Matteucci, F., \& Chiappini, C. 2003, MNRAS, 346, 295

Rood, R. T., Bania, T. W., \& Wilson, T. L. 1992, Nature, 355, 618

Rood, R. T., Bania, T. W., Wilson, T. L., \& Balser, D. S. 1995, in The Light Element Abundances, ed. P. Crane (Berlin: Springer), 201

Shetrone, M. D. 2003, ApJ, 585, L45

Spruit, H. C. 1999, A\&A, 349, 189

Stern, M. E. 1960, Tellus, 12, 172

Talon, S., \& Charbonnel, C. 2004, A\&A, 418, 1051

Tosi, M. 1998, Space Sci. Rev., 84, 207

Tosi, M. 2000, IAUS, 198, 525

Ulrich, R. K. 1972, ApJ, 172, 165

Wade, G. A., Catala, C., Alecian, E., et al. 2007a [arXiv:astro-ph/0701249]

Wade, G. A., Bagnulo, S., Drouin, D., Landstreet, J. D., \& Monin, D. 2007b, MNRAS, 376, 1145

Wolff, S. 1968, PASP, 80, 281

Zahn, J.-P. 1992, A\&A, 265, 115 